reversal in crystallography and its relation to the so called magnetic groups. The symmetries of property tensors up to rank four are fully and explicitly discussed. The reviewer was particularly attracted by the application of the general theory to such phenomena as piezomagnetism, pyromagnetism and the magnetoelectric effects. The experimental evidence for these phenomena, which are 'forbidden' within the framework of the classical (i.e. non-magnetic) point groups, is reviewed.

The article by W. Cochran on lattice vibrations emphasizes theoretical and experimental studies on specific classes of crystals, e.g. covalent crystals, ionic crystals, molecular crystals, and metals. The limitations of the classical Born theory and recent developments (shell model for ionic crystals, non-adiabatic effects in metals) are discussed in some detail against the background of the experimental evidence from X-ray and neutron scattering.

E. Menzel contributes a short article on the preparation and the study of nearly perfect metal surfaces. Its main emphasis is on experimental techniques.

A detailed article by A. C. T. North is devoted to the $\mathrm{X}$-ray crystallography of large molecules of biological importance. A survey of the methods that have been developed in order to solve the phase problem for large molecules and complex structures is followed by a description of the most important examples of structure determinations in biological materials, including nucleic acids, fibrous and globular proteins and some of the viruses. The article is written in a style that will also attract non-specialists.

Finally we mention three contributions of interest to solid-state physicists, namely those on the generation of high magnetic fields (D. B. Montgomery), on the theory of irreversible processes (G. V. Chester) and on the) electronic properties of liquid metals (N. E. Cusack). The last mentioned article is particularly recommended as a presentation of this rapidly developing field that is well balanced between experiment and theory.

Max-Planck-Institut für Metallforschung

A. Segger

7 Stuttgart- $N$

Azenbergstrasse 12

Germany

Glays and clay minerals. Proceedings of the Eleventh National Conference on Clays and Clay Minerals. Edited by W. F. Bradley. Pp. ix +368 . New York: Pergamon Press, 1963. Price $£ 5 \cdot 5 \cdot 0$.

The eleventh annual conference on Clays and Clay Minerals was held in Ottawa, Ontario, on 13-17 August 1962, and this book contains the published proceedings. The main part of the conference was devoted to a symposium on Clay Mineral Transformations at which eighteen papers were presented; sixteen of these are published here in full and abstracts are given of the two others. Almost all the papers are concerned with the mineralogical aspects of weathering. The widespread use of X-ray pow- der diffraction for identification of fine-grained materials is shown by its use in eleven of the sixteen full papers presented in the symposium. The general session contains thirteen papers of which four appear in abstract only. This session covers a wide range of clay-mineralogical topics ranging from a summary of recent work on the cell dimensions of layer-lattice silicates to a paper on clay mineralogy in building research.

The book, as is usual with this annual publication, is well-produced and has a good nine-page index. The editor and the publishers are to be congratulated on reducing the time-lag between conference and publication from nearly two years for the 1960 conference to fifteen months for the 1962 conference. The papers presented at these conferences are of interest to all working on clays and rapid publication greatly enhances the value of the published proceedings.

\section{Rothamsted Experimental Station}

G. Brown

Harpenden

Herts

England

Structure reports for 1953. Volume 17. General Editor, W. B. Pearson and Section Editors W. B. Pearson (Metals), J. Wyart (Inorganic Compounds) and J. Monteath Robertson (Organic Compounds). Pp. viii + 863. Published for the International Union of Crystallography. Utrecht: N. V.A. Oosthoek's Uitgevers Mij, 1963. Price 125 Netherlands Guilders; $\$ 35: £ 12 \cdot 10 s$.

The volume of Structure Reports under review contains the relevant information of crystallographic work published in 1953. Like its predecessors it contains detailed and factual information, concisely presented, of use not only to the specialist but also to those in other disciplines. Nothing that is particularly significant appears to have emerged in 1953 apart from the outstanding contribution towards our understanding the structure of deoxyribonucleic acid, DNA.

These volumes are now under the General Editorship of Dr W. B. Pearson who has, as usual, been assisted by teams of able abstractors. Structures have not been listed according to structure type, as was the case in the original Structurbericht, but in each section in order of increasing complexity. This change has been inevitable in view of the complex types now being revealed in all fields. Naturally this had led to a certain simplicity of approach in the presentation of structures and in the manner of their indexing. As more of these volumes appear and the time gap is narrowed in bringing out back numbers to complete the series it seems inevitable that difficulties will be experienced in tracing, for instance, families of structures through Structure Reports. It will accordingly be very difficult to see in this monumental collection anything but the details that interest the seeker. What is going to be needed therefore is a more comprehensive 
system of classifications with cross-referencing that will allow fuller use to be made of these excellent volumes.

To return to the volume under review: an interesting feature is the emergence of the increasing application of techniques other than the X-ray method to crystallographic problems. For instance, a rapidly developing technique is that of nuclear-magnetic-resonance studies on crystals. One reference is made to it in Volume 13 (1950) whereas eleven cases are mentioned in the present one. The number of neutron-diffraction studies listed in Vol. 17 is more than double that in the previous one and there is almost a proportionate increase in the use of microwave-spectral studies. While such techniques have gone much further in their applications today, it is noticeable that X-ray investigators tend to overlook recording the optical properties of their specimens as revealed by the decline in the numbers of such examinations listed in this volume compared with Vol. 16.

A marked feature of Structure Reports, and not only the present volume, is the tendency to a paucity of adequate structure representations. Surely more could be done to represent completed structures by good crystallographic illustration. There is no uniformity in this respect and this perhaps is a fault not so much of the abstractors as of the authors who could establish better cooperation with abstractors and provide the latter with the relevant illustrations. Such refinements would undoubtedly increase the costs of Structure Reports, but if they are to serve their purpose adequately and not degenerate into mere catalogues some compromise should be sought in the representation of completed structure determinations.

Naturally, the structure determinations in any one field, in one year, do not form a continuity and it would be unfair to single out any particular contribution in a field as diverse as crystallography. It should be realized however that in 1953 Crick \& Watson at Cambridge, Wilkins at King's College, London, and the late Miss Franklin at Birkbeck College, London, were responsible for the major crystallographic break-through that resulted in the structure determination of deoxyribonucleic acid - the carrier of genetic information. In pointing out this single item one is naturally led to ask whether the section editors of Structure Reports could not include brief summaries indicating the significant contributions in any one year. As Structure Reports must of necessity appear in retrospect, this would not be a difficulty imposed on Section Editors, and even if they were accused of personal bias such summaries would still be invaluable to those browsing through the volumes. Here there is scope for imagination and ingenuity in the concise presentation of such articles and would make these valuable volumes all the more attractive.

However, in spite of this minor criticism, both Section Editors and the General Editors are to be complimented on the high standards maintained in the presentation of Structure Reports in a remarkably clear manner. The printing and diagrams, such as they are, are excellent and the volume takes its deserved place with those of its predecessors.

C. H. Carlisle

Birkbeck College Crystallography Laboratory

21 Torrington Square,

London, W. C. 1

England
Theory of elasticity of an anisotropic elastic body. By S. G.LekHnitskir, San Francisco: HoldenDay Inc., 1963. Translated by P. Fern. Edited by J. J. Brandstatter. Pp. 404. Price $\$ 10.95$. Originally published as Teoriia Uprugosti Anisotropnovo Tela. Moscow and Leningrad: Government Publishing House for Technical Theoretical Works.

This is a monograph which treats systematically a number of problems concerned with the elastic deformation of anisotropic bodies. Apart from crystals a number of structures, such as corrugated plates and membranes, which are constructed from isotropic materials are elastically anisotropic. Also reinforced structures containing with a preferred orientation are elastically anisotropic. Thus the work is of importance to missile and aircraft designers, to mining engineers, geophysicists and architectural engineers.

The six chapters deal with (1) the general theory of the elasticity of an anisotropic body, (2) simple cases of elastic equilibrium, (3) and (4) the state of stress of an anisotropic body bounded by a cylindrical surface when the stress is constant along a generator, (5) the state of stress of an anisotropic cantilever deformed by a transverse force, $(6)$ the symmetric deformation and torsion of a body of revolution.

The treatment is at a uniformly high level throughout. The problems are treated purely analytically in a systematic and comprehensive manner. The book is intended for theoreticians rather than practising technologists. There is little direct application of the analysis to actual problems. The translation appears to be excellent and the production of the book has resulted in very clear text, equations and figures.

339 Cherry Hinton Road
Cambridge
England

W. A. Wooster

Kristallchemie. By W. KLeBer. Pp. 128 with many Figs. Leipzig: Teubner. 1963. Price DM 9·30.

The title of this work is misleading, for it is in no sense a systematic presentation of the principles of crystal chemistry. Rather is it a discussion of certain isolated topics in the field, often demanding of the reader a considerable knowledge of structural chemistry for its proper comprehension. Yet the reader with this knowledge will find much that is elementary and much with which he is familiar.

The first chapter (half the book) is devoted to a discussion of the packing of spheres, and it is shown that many structures can be described as a close-packed assembly of atoms of one kind with other atoms in the interstices. Such a description of some structures is undoubtedly illuminating, but there are many others of which the important features are obscured when described in this way: what is significant about the zincblende structure, for example, is not that the zine atoms lie in 